\title{
Modified L'Episcopo Tendon Transfers for Irreparable Rotator Cuff Tears
}

\author{
5-year Followup
}

Christian Gerhardt MD, Lars Lehmann MD, Sven Lichtenberg MD, Peter Magosch MD, Peter Habermeyer PhD

Received: 18 January 2009/Accepted: 27 July 2009/Published online: 15 August 2009

(C) The Association of Bone and Joint Surgeons (B) 2009

\begin{abstract}
Patients with posterosuperior cuff tears lose functional external rotation of the shoulder. Latissimus dorsi and teres major transfer is performed to restore external rotation. Twenty patients with a mean age was $55.8 \pm 6$ years underwent this procedure and were examined at averages of $24.7(\mathrm{n}=17)$ and $70.6(\mathrm{n}=13)$ months. Two patients did not improve presumably because of failure of the transfer. The Constant and Murley score increased from 55.6 to 90.4 after 2 years and to 87.9 after 5 years. The mean active flexion increased from $119.4^{\circ}$ to $169.3^{\circ}$ and reached $170^{\circ}$ after 5 years, and mean external
\end{abstract}

Each author certifies that he or she has no commercial associations (eg, consultancies, stock ownership, equity interest, patent/licensing arrangements, etc) that might pose a conflict of interest in connection with the submitted article.

Each author certifies that his or her institution has approved the human protocol for this investigation, that all investigations were conducted in conformity with ethical principles of research, and that informed consent was obtained.

This work was performed at Atos Klinik, Praxis für Schulter- und Ellbogenchirurgie, Heidelberg, Germany.

L. Lehmann and C. Gerhardt contributed equally to this work.

C. Gerhardt ( $($ )

Charité Universitätsmedizin Berlin, Centrum für muskuloskeletale Chirurgie, Augustenburger Platz 1, 13353 Berlin, Germany

e-mail: christian.gerhardt@charite.de

L. Lehmann

Universitätsmedizin Mannheim, Orthopädisch-

Unfallchirurgisches Zentrum, Mannheim, Germany

S. Lichtenberg, P. Magosch, P. Habermeyer

Atos Klinik, Praxis für Schulter- und Ellbogenchirurgie,

Heidelberg, Germany rotation increased from $12^{\circ}$ to $35^{\circ}$, finally reaching $23^{\circ}$. The grade of cuff arthritis progressed from initially Grade 1 in $17 \%$ and Grade 2 in $28 \%$ to Grade 2 in $8 \%$, Grade 3 in $69 \%$, and Grade 4 in $15 \%$ at final followup. The acromiohumeral distance increased from $4.5 \mathrm{~mm}$ to $6 \mathrm{~mm}$ and decreased to $3.8 \mathrm{~mm}$ after 5 years. Electromyographic analysis showed activity during isometric internal and external rotation in the transferred muscle in all patients. The L'Episcopo procedure can restore shoulder function, but cuff arthropathy may progress.

Level of Evidence: Level IV, therapeutic study. See Guidelines for Authors for a complete description of levels of evidence.

\section{Introduction}

Partial or complete rotator cuff tears occur more frequently with increasing age. Some tears are well tolerated; others cause severe functional limitations and incapacitating chronic pain. If these disorders are caused by massive, irreparable rotator cuff tears, the surgical treatment is still considered controversial and often the results are less favorable and predictable.

The classification of massive cuff tears is not clearly specified. For example, Cofield used the term massive for all tears exceeding $5 \mathrm{~cm}$ [4]. Gerber et al. classified massive cuff tears according to their reparability $[8,11]$. In this classification if the cuff edges cannot be reduced without extensive tension when the arm is abducted to $60^{\circ}$, it is classified as Type III or massive [8, 11]. A later modification of the classification added consideration of the extent of the tear suggesting that lesions involving at least two entire tendons were determined as massive $[9,10]$. 
In 1934, L'Episcopo introduced combined transfer of the tendons of latissimus dorsi and teres major muscle to regain external rotation in obstetric plexus paralysis [17]. Gerber et al. reported that the functional deficits of a posterosuperior cuff tear and the neurologic cuff deficiency resulting from suprascapular nerve lesions seen in brachial plexus palsies are comparable [11]. This concept led the senior author (HP) to perform a modified L'Episcopo technique to restore the loss of external rotation in patients with massive posterosuperior rotator cuff tears. The technique, the original latissimus dorsi transfer described by Gerber et al. [11], used the tendon of the latissimus dorsi to reconstruct the posterior and superior parts of the cuff, through two incisions. In contrast, the modified technique presented in the current study aims to restore the posterior rotator cuff structures thereby restoring external rotation, but it is performed using one incision. Thus, detachment of the deltoid muscle is not required, which reduces the risk of deltoid insufficiency. We suggest the indication for this procedure is loss of external rotation with positive external rotation lag and hornblower sign and considerable loss of active flexion in patients younger than 70 years. A contraindication is impairment of subscapular or deltoid muscle function.

We therefore asked whether the modified L'Episcopo procedure would increase clinical and functional outcomes 2 and 5 years after surgery using the Constant and Murley score [6]. Furthermore, we hypothesize that this technique prevents development of a cuff tear arthropathy as seen on radiologic imaging.

\section{Materials and Methods}

Between 1999 and 2001, 20 consecutive patients underwent transfer of the latissimus dorsi and teres major tendons according to the L'Episcopo technique performed by the senior author (HP).

Data for two patients were excluded because both sustained a fracture of the proximal humerus. Furthermore, one of these patients has an addiction and did not abide by the recommended postoperative restrictions.

The diagnosis was based on clinical examination with evaluation of active ranges of motion, external rotation lag sign, and hornblower sign [21]. Standardized radiographs were performed in three planes to determine cranial migration of the humeral head and grade of osteoarthritis. MRI was performed to evaluate extension and retraction of the cuff tear according to Patte [18] and muscle atrophy using the classification of Thomazeau et al. [20]. Intact subscapular and deltoid muscles were determined by clinical examination and MRI. The clinical examination included the belly-press, belly-off and, if possible, the liftoff test [19], and proper activation of the different portions of the deltoid. Before surgery was elected, all patients attempted but were unsatisfied with nonoperative treatment that consisted of physiotherapy for a minimum of 6 months and nonsteroidal antiinflammatory drugs for pain.

The mean age of the patients at surgery was 55.8 years (range, 34-71 years), with an average duration of clinical symptoms of 30.6 months (range, 6-120 months). One patient had a previous failed open cuff reconstruction.

Surgery was performed with the patient in a lateral decubitus position and the extremity was free-draped. An angled incision was made beginning from the posterior boarder of the deltoid muscle to the axillary fold according to Beauchamp et al. [2] and further medial along the course of the latissimus dorsi muscle. The deltoid muscle was elevated to display the caput longum of the triceps and teres major muscle followed by exposure of the quadrangular space to identify the axillary nerve. Then the latissimus dorsi tendon was identified and the radial nerve was displayed in the triangular space (Fig. 1). The combined insertion of the latissimus dorsi and teres major tendons is observed and sharply dissected from the humerus while taking care to avoid damage to the radial nerve. If necessary, the tendons were sutured together so they did not separate during the procedure. A bony trough was prepared in abduction and external rotation at the lateral proximal humeral shaft. After insertion of four suture anchors, the tendons were attached using \#2 Mersilene sutures (Ethicon, Norderstedt, Germany) with the Mason-Allen suture technique (Fig. 2).

Immediately after surgery, immobilization was accomplished using an abduction pillow for 3 weeks. During this period, passive mobilization was restricted to $30^{\circ}$ abduction, $30^{\circ}$ flexion, $60^{\circ}$ internal, and $0^{\circ}$ external rotation. After the third week, passive range of motion was increased to $60^{\circ}$ abduction, $90^{\circ}$ flexion, and $60^{\circ}$ internal rotation until the end of Week 6 . Then active-assisted and,



Fig. 1 After identifying the insertion of the latissimus dorsi and teres major tendons, they were sharply dissected from the humerus. 


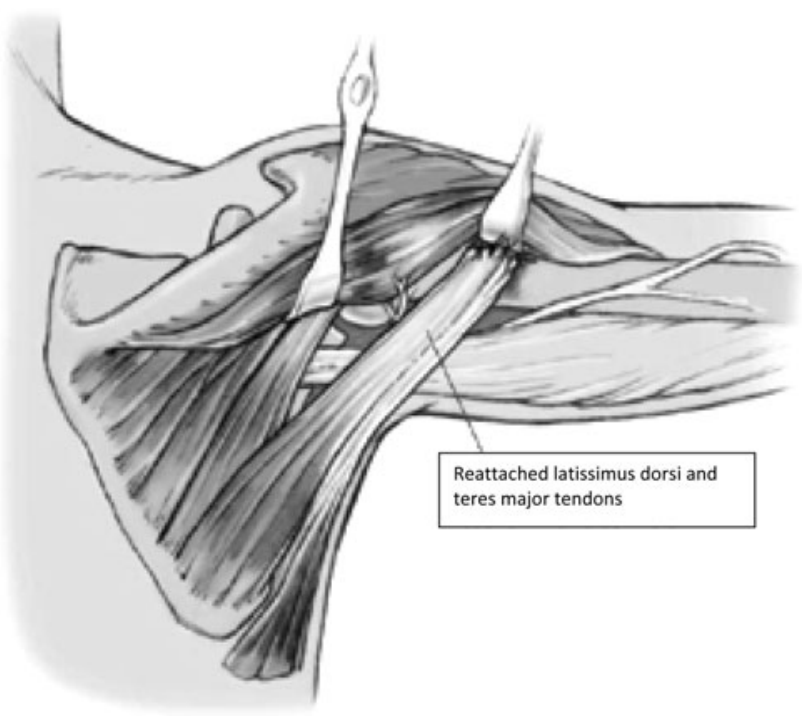

Fig. 2 The transferred tendons were reinserted at the lateral aspect of the humerus.

if possible, active range of motion within a pain-free range was allowed with careful strengthening after the seventh week when full passive mobilization was achieved.

Followups were performed at 13 months (mean, 24.7 months; range, $13-39$ months; $\mathrm{n}=17$ ) and 60 months (mean, 70.8 months; range, 60-87 months; $\mathrm{n}=13$ ). We clinically evaluated the patients using the age- and genderadjusted scoring system according to Constant and Murley [6]. Standard radiography was performed obtaining true anteroposterior, outlet, and axillary views. The acromiohumeral distance was measured in the true anteroposterior view. The grade of glenohumeral osteoarthritis and cuff tear arthropathy was detected using the classification according to Hamada et al. [13]. Clinical and radiologic evaluations were performed by one reviewer (MP).

We verified the integrity of the transferred tendons by ultrasound or MRI. Furthermore, surface electromyography (Noromed MES 9000 surface electromyographic device; Ja Myotronics Noromed Inc, Seattle, WA) was attempted to evaluate activity of the muscle flap at the final followup. This examination was performed in isometric external and internal rotation, abduction, and adduction.

We used SPSS version 13.0 (SPSS Inc, Chicago, IL). The level of significance was set at $p<0.05$. For preoperative and postoperative dichotomous variables such as positive/negative external rotation lag sign or drop arm sign, comparisons were evaluated using the McNemar test (binominal distribution, two-tailed). For comparisons between preoperative and postoperative numerical data such as Constant and Murley scores, range of motion, or strength, the Wilcoxon signed rank test (Monte Carlo, two-tailed), was used.

\section{Results}

The mean Constant and Murley score was improved ( $\mathrm{p}<0.0001$ ) by the reconstruction. The preoperative mean score was 46.5 points (range, 18.0-76.2 points; adjusted Constant and Murley score, $55.6 \% \pm 21.1 \%$ ) and improved to 72.5 points (range, $44.0-91.8$ points; adjusted Constant and Murley score, $90.4 \% \pm 19.9 \%) 2$ years postoperatively and 68.2 points (range, $51.3-80.3$ points; $87.9 \% \pm 12.8 \%$ ) 5 years postoperatively (Fig. 3). The patients showed improvement $(\mathrm{p}<0.0001)$ in parameters such as pain, activities of daily living, active range of motion, and strength of abduction (Table 1). In the Constant and Murley score the strength is measured in $90^{\circ}$ abduction revealing an increase from $(\mathrm{p}<0.05)$ from 1.23 points (range, $0-4.4$ points) to 11.9 points (range, $0-20.8$ points) and declined by the 5-year followup to 5.3 points (range, 2.1-7.7 points). This decline was relatively large, however, the result after 5 years still showed substantial improvement from preoperative strength.

Evaluation of range of motion showed significant increases $(\mathrm{p}<0.0001)$ in active flexion from $119^{\circ} \pm 48^{\circ}$ to $169^{\circ} \pm 25.4^{\circ}$ and $170^{\circ} \pm 7.4^{\circ}$ after 2 and 5 years, respectively. Significant gains in active external rotation $(\mathrm{p}<0.0001)$ were seen from $12^{\circ} \pm 21.1^{\circ}$ to $35^{\circ} \pm 15.4^{\circ}$ after 2 years, but decreased $(\mathrm{p} \geq 0.05)$ to $23^{\circ} \pm 30.5^{\circ}$ at the final followup (Fig. 4). Before surgery, $89 \%$ of the patients had a positive external rotation lag sign, which could be observed in $11.8 \%$ of patients 2 years and $58.3 \%$ of patients 5 years after surgery. A positive drop arm sign was seen in $33.3 \%$ of the patients preoperatively, which resolved postoperatively in all.

Ultrasound and MRI showed consistent integrity of the latissimus dorsi and teres major flap at 2-year and 5-year followups.

Analysis of the radiographs showed a preoperative acromiohumeral distance of $4.5 \mathrm{~mm}$ (range, $2-7 \mathrm{~mm}$ ), which increased to $6 \mathrm{~mm}$ (range, $3-8 \mathrm{~mm}) 2$ years $(\mathrm{p} \geq 0.05)$ after

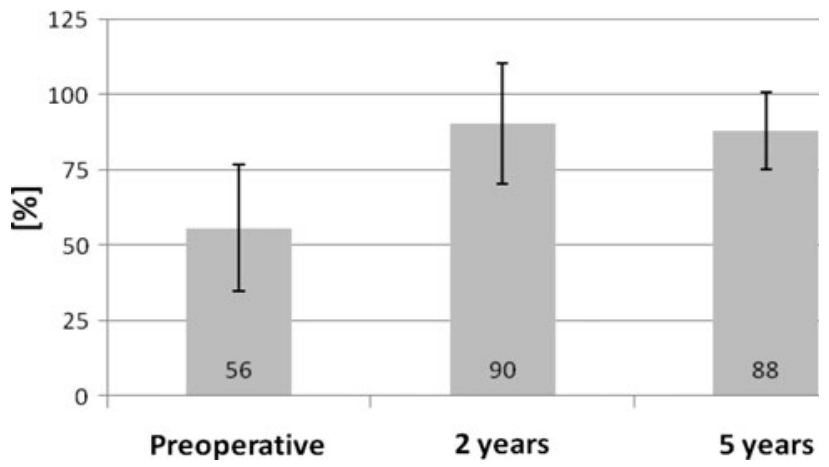

Fig. 3 Age- and gender-adjusted Constant and Murley scores improved during the postoperative period. 
Table 1. Adjusted Constant and Murley scores and range of motion*

\begin{tabular}{llccc}
\hline Times & $\begin{array}{l}\text { Adjusted Constant } \\
\text { \& Murley score (\%) }\end{array}$ & Pain & Elevation (degrees) & $\begin{array}{l}\text { Active external } \\
\text { rotation (degrees) }\end{array}$ \\
\hline Preoperative & $55.6 \pm 21.1$ & $7.5 \pm 4.3$ & $119.4 \pm 48$ & $11.7 \pm 21.1$ \\
2-year followup & $90.4 \pm 19.9^{\dagger}$ & $14.1 \pm 2.1^{\dagger}$ & $169.4 \pm 25^{\dagger}$ & $35.3 \pm 15.4^{\dagger}$ \\
5-year followup & $87.9 \pm 12.8^{\dagger}$ & $13.9 \pm 2.2^{\dagger}$ & $170.0 \pm 7^{\dagger}$ & $22.5 \pm 30.5$ \\
\hline
\end{tabular}

* Significant gain in shoulder function was seen after 2 years, remaining constant with time, except external rotation, which declined; ${ }^{\dagger} \mathrm{p}<0.0001$.

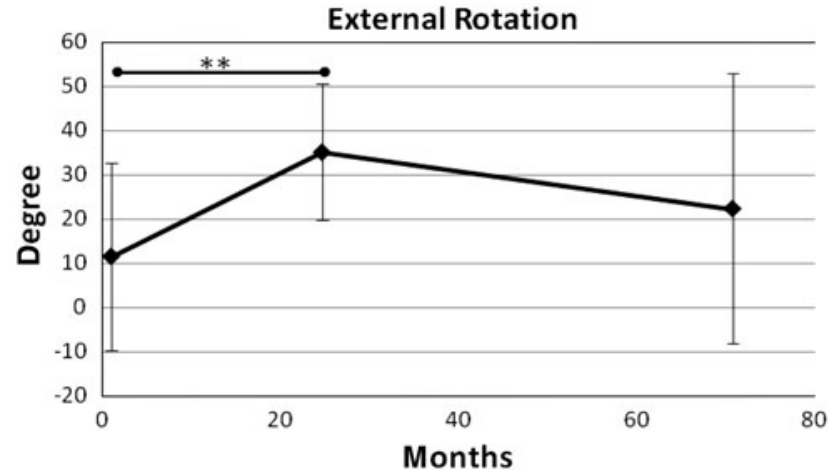

Fig. 4 Active external rotation improves after the tendon transfers but declines over the course of 72 months.

surgery and $3.8 \mathrm{~mm}$ (range, 1-7 $\mathrm{mm}$ ) by 5 years after surgery $(p<0.05)$. Before surgery, $22.2 \%$ of the patients had a cuff tear arthropathy: $11.1 \%$ had Grade 1 and $11.1 \%$ had Grade 2 according to the classification of Hamada et al. [13]. These numbers increased to $23.5 \%$ of the patients with Grade 1 and $17.6 \%$ with Grade 2 arthropathy by 2 years followup. Five years postoperatively, 13\% had Grade 2, $69.2 \%$ had Grade 3, and $15.4 \%$ had Grade 4 cuff tear arthropathy. Averaged, the arthropathy increased from $0.8 \mathrm{~mm}$ initially to $3.1 \mathrm{~mm}$ after 5 years. These changes with time were significant ( $\mathrm{p}<0.001)$. Nevertheless, the increase in osteoarthritis was not associated with a decline in shoulder function as seen in persistently improved Constant and Murley scores.

The ability to learn a new function as analyzed by electromyography using the activity of the transferred muscle flap was apparent in all patients. At followup, all examined patients showed positive findings on resisted external and internal rotation indicating a functional muscle flap. Twenty-seven percent of the patients had higher activity for external than for internal rotation. Seventeen percent had equal activity for external and internal rotation. The other $56 \%$ had higher activity for internal rotation. If the amplitude of electromyographic measurement of external rotation was greater than in internal rotation, this was associated with a higher adjusted Constant and Murley score $(95 \%$, standard deviation \pm 7.8 versus $84.3 \%$, standard deviation \pm 14.5 ).
Except for the two patients mentioned earlier, there were no failures of the transfer or additional complications such as infections, palsies, or bleeding.

\section{Discussion}

In this study, a modified L'Episcopo technique was used to regain external rotation in shoulders with massive posterosuperior tears of the rotator cuff. The 18 patients included had sustained increases in Constant and Murley score during the following years. Furthermore, an important benefit in movement, especially external rotation, was seen postoperatively. However, between the 2-year and 5-year examinations there was an average loss of $23^{\circ}$ external rotation. This was reflected in an increasing number of patients with an external rotation lag sign. Although an initial increase in acromiohumeral distance was seen at the 2-year evaluation, superior migration of the humeral head with concomitant cuff arthropathy was evident by the final 5-year followup.

This study has some limitations. First, we did not perform a randomized investigation comparing the results with an established procedure, eg, the latissimus dorsi transfer, thus a missing control group. Furthermore, two patients were excluded as a result of proximal humerus fractures. In our opinion, this is a second, not foreseeable disorder with major impact to the affected shoulder. We believe this secondary injury complicates interpretation of the results after the tendon transfer. Therefore we felt justified in eliminating these two patients from the final evaluation. Another possible limitation is that only one reviewer, who was not involved in performing the surgeries (MP), did the clinical and radiographic analyses.

The increase in postoperative shoulder function in these patients is similar to the results of other published studies in which the L'Episcopo procedure was used for treatment of brachial plexus injuries, posterosuperior cuff tears, and after latissimus dorsi transfer [2, 3]. Beauchamp et al. described the results of 11 patients undergoing the L'Episcopo procedure resulting from adult-onset brachial plexus injuries with deficient external rotation [2]. The patients had gains in movement, with a mean active flexion 
of $110^{\circ}$ to $173^{\circ}$, and external rotation from $28^{\circ}$ to $69^{\circ}$ postoperatively. Similar results were reported in seven cases of external rotation deficits resulting from posterosuperior cuff tears [3]. After a minimum followup of 15 months (mean, 22 months; range, 15-41 months), shoulder function and motion increased significantly in all cases with a gain of absolute Constant and Murley score of approximately 32 points. The increase in external rotation was approximately $27^{\circ}$ (range, $10^{\circ}-40^{\circ}$ ) with partial or complete decline of drop arm, lag, and hornblower sign. These data are congruent with our findings after 2 years.

For treatment of posterosuperior rotator cuff tears, Gerber et al. [8, 11] and others [1, 12, 16, 23] have described transfer of the latissimus dorsi tendon sutured to the insertion site of the supraspinatus tendon using a twoincision technique.

In 2006, Habermeyer et al. reported data for 14 patients treated with a modified latissimus dorsi transfer using a single-incision technique. The mean Constant and Murley score improved to 74.6 points showing enhancement of pain and an increase in range of motion. The mean active flexion increased from $119^{\circ}$ to $170^{\circ}$, mean abduction from $118^{\circ}$ to $169^{\circ}$, and mean external rotation from $19^{\circ}$ to $33^{\circ}$. Irlenbusch et al. [16] reported continuous improvement in the Constant and Murley score during a 50-month period from 33 points initially to 71 points in patients undergoing primary reconstruction. Range of motion increased with active flexion from $81^{\circ}$ to $166^{\circ}$. The active external rotation increased to approximately $23^{\circ}$. In a sizeable study with a mean followup of 53 months, Gerber et al. reported the results after latissimus dorsi transfer [10]. Substantial increases in the Constant and Murley score and range of motion with a $7^{\circ}$-gain in external rotation were seen.

Taking this marginal gain of external rotation into account, some authors argued combined transfer of latissimus dorsi and teres major tendons might lead to increased restoration of active external rotation resulting from a more powerful transfer [3, 14]. This difference in external rotation might not be permanent as seen with the loss of external rotation between 2 and 5 years in our patients. The cause for this is not fully understood. Possible explanations might be the physiologic decline in muscle strength resulting from maturation [5], or the patient might neglect his or her exercise with time. Still, to our knowledge, no study has evaluated the training effect after a decrease in active external rotation after this procedure.

Data for the latissimus dorsi transfer are comparable to our data regarding pain, function, and range of motion. Furthermore, progression of osteoarthritis and decrease of acromiohumeral distance were reported for the latissimus dorsi transfer [10, 12, 16].
These also were seen in our patients; the initially improved acromiohumeral distance did not persist and the grade of cuff tear arthropathy increased in all patients from an average of $0.8 \mathrm{~mm}$ to $3.1 \mathrm{~mm}$ according to the classification of Hamada et al. [13]. The mean acromiohumeral distance decreased to $3.8 \mathrm{~mm}$ after 5 years.

We did not find clinical evidence on ultrasound examination for failure of the transferred tendons. This is consistent with other studies [1, 3, 10, 12].

Furthermore, we performed electromyographic examination of the activity of latissimus dorsi and teres major muscles during isometric contraction. The results showed clear activity in external and internal rotation movements in all transferred muscles. Particularly, 27\% had a greater activity and $17 \%$ had equal activity for external and internal rotation. Irlenbusch et al. reported a superior increase in Constant and Murley score could be achieved when the transferred muscles have greater activity in external rotation [15]. They concluded the functional improvement was not caused only by an interposition or tenodesis effect. In contrast, Werner et al. observed a concomitant, not selective, signal during rotation in the transferred and contralateral latissimus dorsi muscles [22]. They suggested the muscle might be functioning as a trunk stabilizer and questioned the proposed function of the transferred muscle as a substitute external rotator.

It now is standard practice to perform muscle transfer for treatment of irreparable posterosuperior cuff tears [1, 3, 12]. With these surgeries, satisfactory results can be achieved. Nevertheless, many authors have reported increasing rates of osteoarthritis with an associated decrease of acromiohumeral distance. These findings are consistent with our results. After 5 years, every patient showed degenerative changes of the glenohumeral joint. This was not associated with a lower Constant and Murley score or external/internal rotation ratio on electromyography.

Several factors have been reported to be associated with an inferior functional outcome. Two studies stressed the importance of an intact subscapularis muscle [1, 10]. Furthermore, inferior results with respect to Constant and Murley score, active elevation, and active external rotation can be associated with fatty infiltration of the teres minor muscle [7].

Overall, surgeons can feel confident that, for treatment of massive posterosuperior rotator cuff tears, transfer of latissimus dorsi and teres major tendons or the single latissimus dorsi transfer improves shoulder function especially in external rotation and forward flexion. The combined transfer sometimes is not possible in some patients because the teres major is too short and bulky. This tendon transfer leads to satisfying results and is associated with few complications. Loss of active external rotation 
and an increase in positive external rotation lag signs might appear between 2 and 5 years after surgery. Progression in the radiographic grade of osteoarthritis and decreasing acromiohumeral distance cannot be avoided with the single or combined transfers.

Acknowledgments We thank the team of Atos Klinik, Praxis für Schulter- und Ellbogenchirurgie, Heidelberg, especially Gisela Ludwig, for performing the electromyographic measurements.

\section{References}

1. Aoki M, Okamura K, Fukushima S, Takahashi $\mathrm{T}$, Ogino $\mathrm{T}$. Transfer of latissimus dorsi for irreparable rotator-cuff tears. J Bone Joint Surg Br. 1996;78:761-766.

2. Beauchamp M, Beaton DE, Barnhill TA, Mackay M, Richards RR. Functional outcome after the L'Episcopo procedure. J Shoulder Elbow Surg. 1998;7:90-96.

3. Boileau P, Chuinard C, Roussanne Y, Neyton L, Trojani C. Modified latissimus dorsi and teres major transfer through a single delto-pectoral approach for external rotation deficit of the shoulder: as an isolated procedure or with a reverse arthroplasty. J Shoulder Elbow Surg. 2007;16:671-682.

4. Cofield RH. Subscapular muscle transposition for repair of chronic rotator cuff tears. Surg Gynecol Obstet. 1982;154: 667-672.

5. Constant CR. An evaluation of the Constant-Murley shoulder assessment. J Bone Joint Surg Br. 1997;79:695-696.

6. Constant CR, Murley AH. A clinical method of functional assessment of the shoulder. Clin Orthop Relat Res. 1987; 214:160-164.

7. Costouros JG, Espinosa N, Schmid MR, Gerber C. Teres minor integrity predicts outcome of latissimus dorsi tendon transfer for irreparable rotator cuff tears. $J$ Shoulder Elbow Surg. 2007; 16:727-734.

8. Gerber C. Latissimus dorsi transfer for the treatment of irreparable tears of the rotator cuff. Clin Orthop Relat Res. 1992;275:152-160.

9. Gerber C, Fuchs B, Hodler J. The results of repair of massive tears of the rotator cuff. J Bone Joint Surg Am. 2000;82:505-515.
10. Gerber C, Maquieira G, Espinosa N. Latissimus dorsi transfer for the treatment of irreparable rotator cuff tears. J Bone Joint Surg Am. 2006;88:113-120.

11. Gerber C, Vinh TS, Hertel R, Hess C. Latissimus dorsi transfer for the treatment of massive tears of the rotator cuff: a preliminary report. Clin Orthop Relat Res. 1988;232:51-61.

12. Habermeyer P, Magosch P, Rudolph T, Lichtenberg S, Liem D. Transfer of the tendon of latissimus dorsi for the treatment of massive tears of the rotator cuff: a new single-incision technique. J Bone Joint Surg Br. 2006;88:208-212.

13. Hamada K, Fukuda H, Mikasa M, Kobayashi Y. Roentgenographic findings in massive rotator cuff tears: a long-term observation. Clin Orthop Relat Res. 1990;254:92-96.

14. Herzberg G, Urien JP, Dimnet J. Potential excursion and relative tension of muscles in the shoulder girdle: relevance to tendon transfers. J Shoulder Elbow Surg. 1999;8:430-437.

15. Irlenbusch U, Bernsdorf M, Born S, Gansen HK, Lorenz U. Electromyographic analysis of muscle function after latissimus dorsi tendon transfer. J Shoulder Elbow Surg. 2008;17:492-499.

16. Irlenbusch U, Bracht M, Gansen HK, Lorenz U, Thiel J. Latissimus dorsi transfer for irreparable rotator cuff tears: a longitudinal study. J Shoulder Elbow Surg. 2008;17:527-534.

17. L'Episcopo JB. Tendon transplantation in obstetrical paralysis. Am J Surg. 1934;25:122-125.

18. Patte D. Classification of rotator cuff lesions. Clin Orthop Relat Res. 1990;254:81-86.

19. Scheibel M, Magosch P, Pritsch M, Lichtenberg S, Habermeyer P. The belly-off sign: a new clinical diagnostic sign for subscapularis lesions. Arthroscopy. 2005;21:1229-1235.

20. Thomazeau H, Rolland Y, Lucas C, Duval JM, Langlais F. Atrophy of the supraspinatus belly: assessment by MRI in 55 patients with rotator cuff pathology. Acta Orthop Scand. 1996;67:264-268.

21. Walch G, Boulahia A, Calderone S, Robinson AH. The 'dropping' and 'hornblower's' signs in evaluation of rotator-cuff tears. J Bone Joint Surg Br. 1998;80:624-628.

22. Werner CM, Zingg PO, Lie D, Jacob HA, Gerber C. The biomechanical role of the subscapularis in latissimus dorsi transfer for the treatment of irreparable rotator cuff tears. J Shoulder Elbow Surg. 2006;15:736-742.

23. Zafra M, Carpintero P, Carrasco C. Latissimus dorsi transfer for the treatment of massive tears of the rotator cuff. Int Orthop. 2009;33:457-462. 